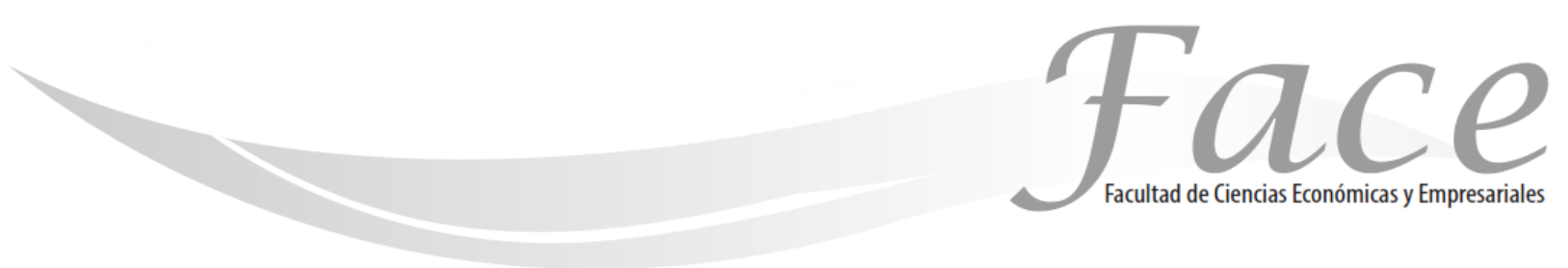

ISSN Impreso: 1794-9920

ISSN Electrónico: 2500-9338

Volumen $18-\mathrm{N}^{\circ} 1$

Año 2018

Págs. 33 - 43

\title{
LA INNOVACIÓN COMO ESTRATEGIA DE SOSTENIBILIDAD Y SU EFECTO EN EL RENDIMIENTO DE LA INDUSTRIA AZUCARERA EN MÉXICO *
}

\author{
Ernesto Cavazos Reyes ** \\ Enlace ORCID: https://orcid.org/0000-0001-6749-5392 \\ Violeta Jiménez Zarate *** \\ Enlace ORCID: https://orcid.org/0000-0003-0257-1125 \\ Gil Santana Esparza **** \\ Enlace ORCID: https://orcid.org/0000-0002-1124-4006
}

Fecha de Recepción: 2 de Mayo 2018

Fecha de Aprobación: 28 de Julio 2018

\section{Resumen:}

México, es un país con gran participación de la industria azucarera, generadora de bienestar económico y social, sin embargo sus procesos contribuyen al deterioro ambiental. El objetivo es analizar la innovación como estrategia de sostenibilidad y su relación en el rendimiento de fábrica. Se analizaron 45 ingenios, a través de la técnica Anova de un factor, comparando el comportamiento de grupos de acuerdo a prácticas sostenibles, los resultados indican que mediante la innovación se logran buenas prácticas de sostenibilidad y aquellos ingenios con alto Índice Sustentabilidad Global (ISG) tienen mayores rendimientos; innovar permite nuevas formas de aportar a la sostenibilidad.

Palabras Clave: Innovación, sostenibilidad, sustentabilidad, rendimiento en fábrica, industria azucarera.

* Artículo resultado de la investigación "La innovación como estrategia de sostenibilidad y su relación con el rendimiento de fábrica de la industria azucarera en México" dentro de la Universidad Autónoma de Tamaulipas, Facultad de Comercio y Administración; iniciado en febrero 2017 y finalizada la primera fase en agosto 2018.

** Licenciado en Sistemas Computacionales, con Maestría en Finanzas y Maestría en Administración. Correo: ecavaso@hotmail.com. Universidad Autónoma de Tamaulipas-FCAT.

*** Ingeniera Industrial, con Maestría en Gestión Administrativa. Correo: violetajimenez18@hotmail.com. Universidad Autónoma de Tamaulipas-FCAT.

**** Ingeniero en Sistemas Computacionales, con Maestría en Comunicación Académica. Correo: gil.santana@itspanuco.edu.mx. Instituto Tecnológico Superior de Pánuco. 


\title{
INNOVATION AS A SUSTAINABILITY STRATEGY AND ITS EFFECT ON THE PERFORMANCE OF THE SUGAR INDUSTRY IN MEXICO
}

\begin{abstract}
:
Mexico is a country with great participation of the sugar industry, generator of economic and social welfare, however its processes contribute to environmental deterioration. The objective is to analyze innovation as a sustainability strategy and its relationship in factory performance. 45 mills were analyzed, through the one-way Anova technique, comparing the behavior of groups according to sustainable practices, the results indicate that through innovation, good sustainability practices are achieved and those mills with a high Global Sustainability Index (ISG) they have higher yields; innovating allows new ways of contributing to sustainability.
\end{abstract}

Keywords: Innovation, sustainability, factory performance, sugar industry.

\section{INOVAÇÃO COMO ESTRATÉGIA DE SUSTENTABILIDADE E SEU EFEITO NO DESEMPENHO DA INDÚSTRIA DO AÇÚCAR NO MÉXICO}

\section{Resumo:}

O México é um país com grande participação da indústria açucareira, gerador de bem-estar econômico e social, porém seus processos contribuem para a deterioração ambiental. $O$ objetivo é analisar a inovação como estratégia de sustentabilidade e sua relação no desempenho fabril. Foram analisadas 45 usinas, através da técnica one-way Anova, comparando o comportamento dos grupos de acordo com práticas sustentáveis, os resultados indicam que através da inovação, boas práticas de sustentabilidade são alcançadas e as usinas com um Índice Global de Sustentabilidade (ISG) elevado eles têm maiores rendimentos; inovar permite novas formas de contribuir para a sustentabilidade.

Palavras chave: Inovação, sustentabilidade, desempenho fabril, indústria açucareira. 


\section{INTRODUCCIÓN:}

Actualmente la dinámica de los mercados ha evolucionado, el aumento de población de acuerdo a la ONU (2015) alcanzó la cifra de 7,300 millones de personas y por ello se requiere de mayor abastecimiento de alimentos. Además, se han modificado las formas de producción y comercialización; la participación global exige innovaciones que permitan maximizar el rendimiento en fábrica de las empresas.

Si bien es cierto que el progreso industrial es benéfico, también es importante destacar que esto conlleva altos costos sociales y ecológicos que contribuyen al cambio climático (FAO, 2017). Derivado del impacto ambiental que ha causado efectos negativos al planeta, la ONU trazo una estrategia de neutralidad climática de las naciones unidas, la cual incluye realizar una valoración de emisiones de gases de efecto invernadero, realizar un esfuerzo por reducir las emisiones y analizar la implicación de costos con miras de alcanzar la neutralidad climática (ONU, 2007). Así mismo, es necesario el cumplimiento de los estándares del total de emisiones producidas por el uso de combustibles fósiles causantes del efecto invernadero (Bonsucro, 2016).

Mucho se ha hablado de los términos sostenibilidad y sustentabilidad, para esta investigación en particular, se tomaran como términos similares, ya que Méndez Chiriboga (2012), señala que la diferencia se encuentra en la ubicación geográfica, porque en Europa se utiliza el termino de sostenibilidad y en América el de sustentabilidad.

De acuerdo a la ONU (1987) con el termino de sostenibilidad y Leff (2000) con el termino de sustentabilidad, hacen énfasis en el compromiso de las generaciones futuras.

Seuring y Müller (2008) y la ONU (2017), señalan que la sostenibilidad posee tres dimensiones económica, social y ambiental; y Martins, Mata, \& Costa (2006) Cortes \& Peña (2015), señalan que la sustentabilidad también posee las mismas tres dimensiones. El interés de esta investigación es ver como las acciones de innovación han generado una clasificación de grupos de acuerdo al nivel de sustentabilidad, el cual tiene influencia en el rendimiento de fábrica, pero al contextualizarlo en México solo se ha encontrado literatura que hace referencia a la sustentabilidad en la industria azucarera, en la cual AguilarRivera (2005) considera los elementos de los tres ejes para la construcción del Índice de sustentabilidad global, el cual será considerado como una variable categórica, con el fin de organizar en grupos, de los cuales se analizará a través del Anova de un factor la variable numérica de rendimiento en fábrica.

Existe evidencia empírica, que indica que las producciones relacionadas con el sector azucarero han provocado efecto negativos al ambiente debido a la generación de residuos materiales y energéticos, esto ha inducido a un cambio de legislaciones más severas, lo que lleva a las industrias a una búsqueda por implementar mejores prácticas que ayuden a la sostenibilidad (González-Cortés, GonzálezSuárez, González-Morales, \& Albernas-Carvajal, 2013). La globalización nos invita a desarrollar innovaciones para productos y procesos productivos (Torres-Vargas \& JassoVillazul, 2014). La innovación en procesos tales como: la introducción de nuevas tecnologías, la optimización de procesos y la disminución de vapor; permite a la industria azucarera procesos productivos más limpios (GonzálezCortés et al., 2013), los cuales contribuyen a lograr niveles más altos en el índice de sustentabilidad global ISG. Además, la innovación orientada a la sostenibilidad también considera un cambio de filosofía y valores de la organización (Adams, Jeanrenaud, Bessant, Denyer, \& Overy, 2016), del mismo modo, Barbieri, Marques, Schoproni, Maffini, \& Zamberlan, (2013), señalan que estas dos variables son esenciales en el ámbito empresarial. La innovación permite una transición más eficaz hacia la sostenibilidad (El Bilali, 2018). Estas innovaciones son la base de la sostenibilidad, la cual se traducirá en un mayor rendimiento en fábrica de la industria azucarera.

El objetivo es analizar la innovación como estrategia de sostenibilidad y su relación con el rendimiento de fábrica de la industria azucarera en México.

Las preguntas de investigación son: ¿Cuál es el papel de la innovación como estrategia de sostenibilidad y su efecto en el rendimiento de fábrica de la industria azucarera en México? ¿Los ingenios con alto ISG tienen mayor rendimiento en fábrica?

Los puntos a analizar son la innovación, sostenibilidad y el rendimiento en fábrica de la industria azucarera, de acuerdo a los requerimientos de comercialización internacional, cada vez son más las empresas que se vuelven sostenibles al manejar buenas prácticas ambientales, sociales y económicas.

Este trabajo se integra por cinco apartados: en la primera parte de introducción, se realiza el planteamiento del problema, en este caso se fundamenta él porque es importante el tema de investigación, se traza el objetivo y preguntas de investigación; en la segunda parte se desarrolla con base teórica las generalidades de la industria azucarera, la innovación, sostenibilidad y 
rendimiento de fábrica, en la tercera se define la metodología a utilizar para el estudio, en la cuarta se presentan los resultados del tratamiento de datos, en la quinta parte se plantan las conclusiones y futuras líneas de investigación, además se agrega la bibliografía.

\section{MARCO REFERENCIAL:}

Los ingenios azucareros mexicanos datan de la época colonial, y han evolucionado a lo largo del tiempo, desde entonces han sido fuente de bienestar económico y social, además la caña de azúcar es el cultivo agrícola más importante según la UNESCO (ZAFRANET, 2018). Este análisis toma como base la Teoría de Capacidades Dinámicas de Teece y Pisano (1994), en el sentido de que las empresas logran altos rendimiento debido a las capacidades que tiene como organización para lograr mejoras en sus procesos, resaltando que esas capacidades son construidas a través de la experiencia, dando paso a la innovación. A continuación se presentan las generalidades de la industria azucarera, la innovación, sostenibilidad y el rendimiento de fábrica.

\section{Generalidades de la industria azucarera.}

La industria azucarera, es muy relevante en México, con 51 ingenios instalados (CONADESUCA, 2017b), es el sexto productor de caña de azúcar del mundo, teniendo presencia en 15 estados, genera 450 mil empleos directos, en la zafra 2015-2016 alcanzo, el 0.47 por ciento del Producto Interno Bruto (PIB) general y el 9\% del PIB (SAGARPA, 2016). En este mismo periodo alcanzo una producción de 6,056 millones de toneladas de azúcar (CONADESUCA, 2016). Debido a su gran impacto económico, la industria azucarera requiere de la innovación para poder adoptar mejores prácticas para lograr la sostenibilidad, la cual permitirá consolidar la empresa a largo plazo (Bonsucro, 2016). La innovación da como resultado la ventaja competitiva (Jasso, 2010), actualmente las empresas requieren de innovación, la cual contribuye en gran medida a la sostenibilidad (Mousavi \& Bossink, 2017). La innovación organizacional, de productos y procesos se traducen en mayor rendimiento para las empresas (Camisón \& Villar-López, 2014). Para centrar el estudio en las bases científicas se consideró la teoría de capacidades dinámicas, los cuales favorecen el crecimiento empresarial (Teece \& Pisano, 1994).

\section{Innovación.}

Desde la aparición del hombre, han existido cambios propios de la evolución, los cuales han sido representativos de su época y existen registros de tales hechos históricos, permitiendo la clasificación en órdenes mundiales para iniciar con el descubrimiento de américa, como el 1er. Orden esta la revolución industrial, en el 2do Orden se da el desarrollo de los ferrocarriles, en el 3er. Orden es la era de la electricidad, en el 4to. Orden lo representa la producción fordista y actualmente el 5to. Orden es la sociedad del conocimiento (Jasso, 2005). En todos los casos la única constante es el cambio o bien a lo que llamamos innovación. El proceso de cambio se genera a partir de ciclos, los cuales requieren la tecnología, es ahí donde surgen nuevas oportunidades para los países latinoamericanos (Pérez, 1986). Debido a las diferencias existentes en países desarrollados y los de vías de desarrollo, existe más dificultad de integrar los factores necesarios para que se den los círculos que propician la innovación, estudiosos han realizado un mapeo de américa latina respecto a la posición mundial (Fajnzylber, 1989).

En este sentido es importante destacar que las empresas juegan un papel relevante en el desarrollo y se centran en atender a tres dimensiones: económico, social y ambiental (Seuring \& Müller, 2008), para mejorar el rendimiento se deben aprovechar las capacidades internas para fomentar la innovación (Poorkavoos, Duan, Edwards, \& Ramanathan, 2016). Las empresas van acumulando conocimientos, capacidades y recursos, los cuales les permiten consolidares y crecer a lo largo del tiempo, es importante conocer la trayectoria tecnológica, ya que ella marca la evolución de las empresas y presenta tres etapas: innovadora, estancamiento y obsolescencia; su medición es interesante ya que se asocia con la dinámica de producción y la del mercado (Jasso-Villazul, 2004).

La innovación es producto de la colaboración y aprendizaje, pero son las empresas quienes realizan mayores inversiones en I + D (Jasso, 1998) y con ello generan productos sostenibles (Dangelico, Pujari, \& Pontrandolfo, 2017). Estudios sugieren que el uso de tecnologías favorece la innovación de procesos en las empresas ya que fomenta la I + D (Sun, Hou, \& Li, 2017). La relevancia de la innovación tecnológica incluye los avances en conocimientos y la mejora continua en productos y procesos o bien los cambios profundos en las técnicas de producción (Jasso, 2004).

El núcleo central de la innovación se compone por el comportamiento estratégico, la alianza de empresas y la interrelación: empresa-centros de investigación-universidad (Torres-Vargas \& Jasso-Villazul, 2014). En la innovación se identifican las capacidades organizaciones y gerenciales las cuales son la base para las capacidades dinámicas (Mousavi \& Bossink, 2017). Así se debe incentivar la innovación en los gerentes para que resuelvan los problemas de sostenibilidad (Adams et al., 2016). Las 
innovaciones en los procesos productivos y productos, dan pie a la sostenibilidad.

\section{Sostenibilidad.}

La sostenibilidad permite identificar y evaluar los impactos ambientales que originan las empresas, así como establecer los planes de contingencias que permitan operar de manera amigable con el medio ambiente (Mousavi \& Bossink, 2017).

La ONU en Commission on Environment (1987), define a la sostenibilidad como: "Satisfacción de las necesidades de la generación presente sin comprometer la capacidad de las generaciones futuras para satisfacer sus propias necesidades".

Para lograr la sostenibilidad, es necesario adoptar nuevas formas de producción. Dentro del crecimiento natural de las empresas se observa que entre más se va consolidando la empresa, mayor será la complejidad de la estructura y se requiere mejorar las capacidades tecnológicas y emprendedoras (Torres \& Jasso, 2017). Esa mejora que incluye la búsqueda, desarrollo y adopción de nuevas formas de pensamiento y ejecución de tareas al interior de las organizaciones es: la innovación (Jasso, 2010). Según Tsai \& Liao (2017), las empresas que de manera voluntaria adoptan sistemas sostenibles, proyectan una mejor imagen corporativa. Por otro lado, Bossink (2012), conceptualiza la innovación sostenible como los esfuerzos de las empresas "para mantener, mejorar y renovar la calidad ambiental, social y económica de sus procesos comerciales y los productos y servicios que estos procesos comerciales producen".

La temática ha alcanzado proporciones mundiales, al grado que la FAO ha determinado que: "Nuestros sistemas de explotación agrícola derivan de prácticas insostenibles que han despilfarrado los recursos naturales, dejando a las generaciones actuales y futuras la tarea adicional de abordar la degradación del suelo, la escasez y contaminación del agua, el deterioro de la agrobiodiversidad y el cambio climático" (FAO, 2017) . En un futuro solo aquellas empresas que apostaron por tener la sostenibilidad como ventaja competitiva permanecerán en el mercado (Nidumolu, Prahalad, \& Rangaswami, 2009).

En búsqueda de una producción sostenible de la caña de azúcar se conforma Bonsucro, que consiste en una iniciativa global para el ámbito empresarial, con la finalidad de crear productos más amigables con el medio ambiente, respetando los derechos humanos y condiciones laborarles, permitiendo que el negocio sea productivo y perdure en el tiempo, con ello busca implementar buenas prácticas agrícolas e industriales. Haciendo un análisis de los tres pilares que lo integran social, ambiental y económico se encuentra lo siguiente: 1) enfoque social: se encarga de ramas como salarios, trabajos forzosos, discriminación, equidad de género, días de descanso, horas laborales por semana, cumplimiento legal, sueldos y seguridad para los trabajadores, erradicación del trabajo infantil, entre otras cosas; 2) enfoque ambiental: aquí se ven los temas de manejo de agua, manejo de residuos, control de emisiones, etc. y 3 ) enfoque económico: en este aspecto se analiza la productividad, ganancias, mejora continua, sistemas de gestión y rentabilidad (Bonsucro, 2016). La responsabilidad de cumplir con las disposiciones legales y morales corresponde a los dueños, administradores y a la comunidad en general (Jasso, 2016).

\section{Rendimiento de fábrica.}

El rendimiento en fábrica en la zafra 2015/16 alcanzó un valor promedio de 11.158, de acuerdo a los datos obtenidos de todos los ingenios en México (CONADESUCA, 2016). La producción de azúcar de caña es de 47243071 (miles de pesos) los cuales se dividen en: 3075311 (miles de pesos) de azúcar de blanco especial, 24582204 (miles de pesos) de azúcar estándar, 273750 (miles de pesos) de mascabado, 17169877 (miles de pesos) de azúcar refinada y 2141929 (miles de pesos) de otros productos (Instituto Nacional de Estadistica y Geografía, 2014).

Para incrementar el rendimiento de fábrica, es necesario atender diversos elementos. La competitividad de una industria es resultado de esfuerzos, estrategias y acciones que se realizan por la influencia del medio en que se encuentra (Jasso Villazul, 1997). De acuerdo a Carlos Rello, México exporta 1.2 millones de toneladas de azúcar a estados unidos (ZAFRANET, 2016). Los productos que son más innovadores, tienen mayores oportunidades en mercados internacionales (Jasso Villazul, 1997). Para Lee \& Zhang (2017), la innovación y la sostenibilidad permiten mayores rendimientos. La integración de capacidades, el establecimientos de rutinas que permiten homogeneizar las actividades y definir procesos estandarizados, ha permitido la evolución en el pensamiento del personal, a través del cumulo de conocimientos adquiridos a través de la experiencia y del desarrollo o uso de tecnologías; estas capacidades de acuerdo a estudios empíricos ha permitido el aprendizaje e innovación en compañías con presencia internacional (Torres Vargas \& Jasso Villazul, 2016).

\footnotetext{
Hipótesis.

H1: La innovación como estrategia de sostenibilidad tiene un efecto diferenciador en la media de los grupos de acuerdo al Índice de Sustentabilidad Global de los Ingenios en México.

H2: El rendimiento en fábrica es mayor en el grupo con ISG alto, que en los grupos medio y bajo de la industria azucarera en México.
} 


\section{METODOLOGÍA:}

El análisis se realiza a través de la metodología cuantitativa, mediante la técnica Anova de un factor. De manera inicial se consideran los 51 ingenios instalados en México, descartando aquellos que no cumplen con los datos completos (3 ingenios) y también los que presentan outliers ( 3 ingenios) y se finaliza con una muestra de 45 ingenios. Para este análisis se considera que "la zafra es la fabricación del azúcar de caña, y, porext., del de remolacha ", Tiempo que dura la zafra (Real Academia de la lengua, 2017). Es decir, la zafra es el periodo de tiempo en el que se realiza el proceso de elaboración de azúcar en los ingenios azucareros. En ese sentido, considerando el rendimiento en fábrica del período Zafra 2015/16, información obtenida de la base de datos de CONADESUCA (2016), se analizan los datos en IBM SPSS Statistics 20, con el fin de realizar un comparativo sobre el efecto de la sostenibilidad como estrategia de innovación en el rendimiento de fábrica de la industria azucarera de acuerdo al semáforo del ISG en donde se clasifica en Alto, Medio y Bajo; para el tratamiento de datos se efectúan los estadísticos descriptivos, identificando los outliers los cuales se retiran para homogeneizar los datos, así mismo se aplica la prueba de normalidad de kolmogorov-smirnov y finalmente se aplica la prueba paramétrica, ANOVA de un factor, en donde el ISG es una variable categórica y el rendimiento en fabrica una variable numérica.

\section{RESULTADOS:}

Procedimiento: se realizaron los estadísticos descriptivos y se descartaron 3 ingenios por que la información estaba incompleta, 2 no contaban con el resultado del semáforo del ISG y 1 no tenía el rendimiento en fábrica, quedando 48 ingenios. Se detectaron outliers que corresponden a 3 ingenios, los cuales presentan valores muy dispersión en relación a la media del ISG. Los resultados del Box Plot una vez detectados los outliers se descartaron, quedando 45 ingenios para el análisis, ver tabla 1.

Tabla 1. Estadísticos descriptivos

\begin{tabular}{l|c|c|c|c|c}
\hline & N & Mínimo & Máximo & Media & $\begin{array}{c}\text { Desv. } \\
\text { típ. }\end{array}$ \\
\hline $\begin{array}{l}\text { ZAFRA1516 } \\
\text { N válido } \\
\text { (según lista) }\end{array}$ & 45 & 9.4400 & 12.8300 & 11.128222 & .8477062 \\
\hline
\end{tabular}

Fuente: Elaboración propia a partir de datos de Zafra 2015/16 en SPSS (Conadesuca, 2016).
Tabla 2.

Comparación de medias.

\begin{tabular}{l|l|r|r}
\hline ISG & Media & N & \multicolumn{1}{c}{ Desv. típ. } \\
\hline ALTO & 11.753571 & 14 & .4334052 \\
MEDIO & 10.955714 & 14 & 1.0270249 \\
BAJO & 10.755294 & 17 & .6712313 \\
Total & 11.128222 & 45 & .8477062 \\
\hline
\end{tabular}

Fuente: Elaboración propia a partir de datos de Zafra 2015/16 en SPSS (Conadesuca, 2016).

Tabla 3.

Prueba de normalidad Kolmogorv- Smirnov.

\begin{tabular}{l|r|r|r|r|r|r}
\hline & \multicolumn{3}{|c|}{ Kolmogorov-Smirnova } & \multicolumn{3}{c}{ Shapiro-Wilk } \\
\cline { 2 - 7 } & Estadístico & gl & Sig. & Estadístico & gl & Sig. \\
\hline ZAFRA1516 & .097 & 45 & $.200^{*}$ & .970 & 45 & .283 \\
\hline
\end{tabular}

*. Este es un límite inferior de la significación verdadera.a. Corrección de la significación de Lilliefors

Fuente: Elaboración propia a partir de datos de Zafra 2015/16 en SPSS (Conadesuca, 2016).

Después de retirar los datos atípicos, el análisis comprende los datos de 45 ingenios que cumplen con las características para continuar en la muestra, como $n>30$ se calculó la media de los grupos tal como se muestra en la Tabla 2 observando que el ISG alto posee un valor mayor; después en la Tabla 3 se aplicó, la prueba de Kolmogorv- Smirnov, de acuerdo a los datos obtenidos El valor $p$ es $>.05$ No se rechaza $\mathrm{H} 0$, por lo que existe normalidad en los datos. A continuación se realizó la prueba ANOVA de un factor, en la Tabla 4, se observa la significancia menor a 0.005 lo cual da validez a los resultados, además se observa un valor $\mathrm{F}$ considerable lo que significa que las medias de la variable dependiente difieren entre los grupos de la variable independiente.

Tabla 4.

Anova de un factor

\begin{tabular}{l|r|r|r|r|r}
\hline & \multicolumn{1}{|c|}{$\begin{array}{c}\text { Suma de } \\
\text { cuadrados }\end{array}$} & gl & $\begin{array}{c}\text { Media } \\
\text { cuadrática }\end{array}$ & $\mathrm{F}$ & Sig. \\
\hline Inter-grupos & 8.256 & 2 & 4.128 & 7.421 & .002 \\
Intra-grupos & 23.363 & 42 & .556 & & \\
Total & 31.619 & 44 & & & \\
\hline
\end{tabular}

Fuente: Elaboración propia a partir de datos de Zafra 2015/16 en SPSS (Conadesuca, 2016) 
Tabla 5.

Comparaciones entre medias de grupos.

\section{Comparaciones múltiples}

Variable dependiente: ZAFRA1516 HSD de Tukey

\begin{tabular}{|c|c|c|c|c|c|c|}
\hline \multirow[t]{2}{*}{ (I) ISG } & \multirow[t]{2}{*}{ (J) ISG } & \multirow{2}{*}{$\begin{array}{c}\text { Diferenci } \\
\text { a de } \\
\text { medias } \\
(I-J)\end{array}$} & \multirow[t]{2}{*}{$\begin{array}{l}\text { Error } \\
\text { típico }\end{array}$} & \multirow[t]{2}{*}{ Sig. } & \multicolumn{2}{|c|}{$\begin{array}{c}\text { Intervalo de } \\
\text { confianza al 95\% }\end{array}$} \\
\hline & & & & & $\begin{array}{l}\text { Limite } \\
\text { inferior }\end{array}$ & $\begin{array}{l}\text { Límite } \\
\text { superior }\end{array}$ \\
\hline \multirow{2}{*}{ ALTO } & $\begin{array}{l}\text { MEDI } \\
0\end{array}$ & $.7978571^{*}$ & $\begin{array}{r}281896 \\
4\end{array}$ & $\begin{array}{r}.01 \\
9\end{array}$ & .112991 & $\begin{array}{r}1.48272 \\
3\end{array}$ \\
\hline & BAJO & $.9982773^{*}$ & $\begin{array}{r}269172 \\
7\end{array}$ & .00 & .344324 & 1.65223 \\
\hline \multirow[t]{2}{*}{ MEDIO } & ALTO & $.7978571^{*}$ & $\begin{array}{r}281896 \\
4\end{array}$ & $\begin{array}{r}.01 \\
9\end{array}$ & $\begin{array}{r}1.48272 \\
3\end{array}$ & -112991 \\
\hline & BAJO & .2004202 & $\begin{array}{r}269172 \\
7\end{array}$ & $\begin{array}{r}.73 \\
9\end{array}$ & -.453533 & .854374 \\
\hline \multirow{2}{*}{ BAJO } & ALTO & $.9982773^{*}$ & $\begin{array}{r}269172 \\
7\end{array}$ & $\begin{array}{r}.00 \\
2\end{array}$ & $\begin{array}{r}1.65223 \\
1\end{array}$ & -344324 \\
\hline & $\begin{array}{l}\text { MEDI } \\
0\end{array}$ & .2004202 & $\begin{array}{r}269172 \\
7\end{array}$ & $\begin{array}{r}.73 \\
9\end{array}$ & -854374 & .453533 \\
\hline
\end{tabular}

*. La diferencia de medias es significativa al nivel .05 .

\begin{tabular}{c|r|r|c}
\multicolumn{3}{|c}{ ZAFRA1516 } \\
\cline { 3 - 4 } HSD de Tukey & $\mathrm{N}$ & \multicolumn{2}{|c}{ Subconjunto para alfa $=.05$} \\
\cline { 3 - 4 } ISG & & 1 & 2 \\
& & 10.755294 & \\
\hline BAJO & 17 & 10.955714 & 11.753571 \\
MEDIO & 14 & .746 & 1.000 \\
ALTO & 14 & \\
Sig. & &
\end{tabular}

Se muestran las medias para los grupos en los subconjuntos homogéneos.

a. Usa el tamaño muestral de la media armónica $=14.875$.

b. Los tamaños de los grupos no son iguales. Se utilizará la media armónica de los tamaños de los grupos. Los niveles de error de tipo I no están garantizados.

Fuente: Elaboración propia a partir de datos de Zafra 2015/16 en SPSS (Conadesuca, 2016).

En la tabla 5, de comparaciones entre medias de grupos, se aprecia que en la primera estimación de medias, por grupos clasificando aquellas que tienen un ISG alto, medio y bajo, se encontró que los ingenios con alto ISG, muestran mayor rendimiento en fábrica. Se hace énfasis en que el logro de valores altos de ISG está dado por las innovaciones utilizadas como estrategia para alcanzar la sostenibilidad. Derivado del análisis ANOVA de un factor se obtuvo lo siguiente, que existen valores $p<.05$ (resaltados en cuadros rojos) lo que indica que se rechaza la hipótesis nula $\mathrm{HO}$, la cual plantea una igualdad de medias entre los tres grupos ( $\mathrm{HO}: \mu$ ISG Alto $=\mu$ ISG Medio $=\mu$ ISG Bajo) y no rechaza la hipótesis alternativa $\mathrm{Ha}$, la cual sugiere diferencia de medias (Ha: $\mu$ ISG Alto $\neq \mu$ ISG Medio $\neq \mu$ ISG Bajo), estos valores son los siguientes: diferencia entre $\mu$ ISG Alto y $\mu$ ISG Medio; diferencia entre $\mu$ ISG Alto y $\mu$ ISG Bajo; igualdad entre $\mu$ ISG Medio y $\mu$ ISG Bajo. Dicho análisis se determinó con un nivel de confianza del $95 \%$, contrastando con los valores del rendimiento en fábrica de los Ingenios en México.

Retomando las hipótesis de investigación, se tiene H1: La innovación como estrategia de sostenibilidad tiene un efecto diferenciador en la media de los grupos de acuerdo al Índice de Sustentabilidad Global de los Ingenios en México. De acuerdo a los resultado obtenidos se puede observar que existe diferencia de medias en los casos siguientes: la media del rendimiento de fábrica de los ingenios con alto índice de sostenibilidad la cual es usada como innovación en los proceso de elaboración es diferente de los ingenios con ISG medio y bajo. También se encontró igualdad de medias en el rendimiento de fábrica en ingenios con ISG medio y bajo.

Por tanto, también se da respuesta a la segunda hipótesis: H2: El rendimiento en fábrica es mayor en el grupo con ISG alto, que en los grupos medio y bajo de la industria azucarera en México. La innovación es fundamental para mejorar, de acuerdo de este análisis de la zafra 2015/16, se puede concluir que los ingenios que han implementado innovaciones en sus procesos y han logrado obtener un ISG Alto (Quesería, San Francisco Ameca, José Ma. Martínez (Tala), Lázaro Cárdenas, La Gloria, El Molino, Santa Clara, Puga, Tamazula, Bellavista, Pedernales, Atencingo, Melchor Ocampo y Pujiltic (Cía. La Fe), tienen una media superior en el rendimiento en fabrica sobre aquellos que tienen un ISG Medio (San Nicolás, San Miguel Del Naranjo, El Modelo, El Potrero, Plan De San Luis, El Dorado, Central Progreso, Pánuco, Tres Valles, Huixtla, Aaron Saénz Garza, El Mante, El Higo y, Mahuixtlan) y Bajo (Constancia, San Rafael De Pucté, Santa Rosalía, San Miguelito, Presidente Benito Juárez, La Joya, José Ma. Morelos, Pablo Machado, Central Motzorongo, El Refugio, San José de Abajo, Adolfo López Mateos, San Pedro, Alianza Popular, Cuatotolopam, Plan De Ayala y San Cristóbal).

\section{CONCLUSIONES:}

Desde sus inicios la industria azucarera en México ha sido considerada como uno de los ejes principales de la economía, a través de los años ha sido incentivada y apoyada para su crecimiento y sostenibilidad a través del tiempo. En el último siglo está industria ha despegado de 
ISSN: 1794-9920 - ISSN Electrónico: 2500-9338

Enero - Julio de 2018

Volumen 18 Número 1, Año 2018 Págs. 33 - 43

manera exponencial su productividad y rendimientos con los cuales hoy en día se sitúa en el sexto lugar de producción a nivel mundial.

En ese sentido y en relación al objetivo general planteado en la presente investigación que se orientó a analizar la innovación como estrategia de sostenibilidad y su relación con el rendimiento de fábrica de la industria azucarera en México, se puede decir que el camino para lograr el posicionamiento actual de la industria azucarera en México no ha sido sencillo, pero si fructífero. El cumulo de estrategias que se han implementado con el paso de los años ha aportado a la industria mayores soportes para su desarrollo. Una de las estrategias que en estos últimos años se ha aportado a la industria del azúcar es la Innovación. La innovación como estrategia de sostenibilidad económica ha logrado que los grupos azucareros e ingenios particulares logren dar el empuje que los posicione en los primeros lugares de producción.

Con base a los resultados obtenidos y derivados de la pregunta de investigación, en cuanto al papel de la innovación como estrategia de sostenibilidad y su efecto en el rendimiento de fábrica de la industria azucarera en México, se llega a la conclusión de que el tema de innovación en la industria actualmente está enfocado a la diversificación de subproductos ya que un buen número de ingenios están apostando al rubro de la cogeneración de energía limpia a base de bagazo de la caña. Para hacer esto posible los ingenios en México tienen que hacer uso de nuevas tecnologías y conocimientos internacionales para aprovechar infraestructuras actuales desde el enfoque industrial e implementar nuevos equipos como son: calderas de alta presión y turbogeneradores de alto poder de generación. Actualmente en México se cuenta con 10 ingenios que hicieron sus ajustes para poder implementar la cogeneración y venta de energía eléctrica limpia a la red eléctrica del gobierno federal. Esto es un claro ejemplo de la búsqueda de alternativas con el uso de la innovación para el establecimiento de la sostenibilidad económica y ambiental de la industria azucarera mexicana. Con los resultados de la aplicación de la innovación se obtienen mayores beneficios en rendimientos y eficiencias a través de la cadena de valor del ingenio.

La innovación en la industria azucarera no solo es aplicada en el área industrial, sino que también para el enfoque agrícola se obtienen beneficios en rendimientos impactando la sostenibilidad económica, así como reducción en impactos ambientales y sociales.

La innovación en el área agrícola es aplicada a base de la implementación de tecnología en agricultura de precisión para la preparación de tierras, aplicación de fertilizantes y herbicidas, riego agrícola y el manejo de la cosecha de caña en verde utilizando cosechadores de altos rendimientos de corte de caña.

Otros de los enfoques de innovación aplicada en los campos agrícolas en la creación y diseminación de biofertilizantes, bioherbicidas, manejo de controles biológicos para plagas, mejoramiento de variedades de caña con aumentos de rendimientos y mayores potenciales para contrarrestar enfermedades.

La aplicación de las tecnologías y la innovación para el campo en la industria azucarera ha acarreado beneficios que impactan directamente en la sostenibilidad económica al traer a primer plano el mejoramiento en ingresos y ahorros para la producción de la caña de azúcar, así como ahorros en las cosechas y aumentos en rendimientos de caña por hectárea y azúcar por hectárea. Las innovaciones en el impacto de la sostenibilidad ambiental han reducido las emisiones de gases de efecto invernadero al implementar equipos para la cosecha en verde y se evita la quema de la caña, mejoramiento en el uso del agua y aplicación de fertilizantes y herbicidas amigables con el medio ambiente. La innovación en el impacto social mejoramientos en el cuidado de los trabajadores y establecimientos de mecanismos para respuestas inmediatas a accidentes y control de riesgos utilizando mejores herramientas y tecnología en los campos y equipos de instrumentación en las fábricas.

Se demostró que la innovación como estrategia de sostenibilidad tiene un efecto diferenciador en la media de los grupos de acuerdo al Índice de Sustentabilidad Global de los Ingenios en México. Además que el rendimiento en fábrica es mayor en el grupo con ISG alto, que en los grupos medio y bajo de la industria azucarera en México. Se considera que el acceso a la innovación como estrategia en la industria del azúcar en México ha contribuido fuertemente para ir logrando poco a poco la sostenibilidad de esta industria a través de los años y que no sea vista como una novedad, sino que forme parte de la cultura organizacional en toda su cadena de suministro.

La innovación ha permitido modificar los productos y procesos, en el caso de la industria azucarera, la tendencia es ser empresas sostenibles y se tienen las siguientes oportunidades para futuras investigaciones para demostrar que los ingenios que poseen una alto índice de sostenibilidad global, han atendido acertadamente las tres dimensiones: i) la sostenibilidad económica alta, otorga más rentabilidad a largo plazo, ii) la sostenibilidad ambiental alta, genera menores impactos en suelo y aire y iii) la sostenibilidad social alta, trae mayor inclusión y bienestar social.

También, se puede trabajar realizando análisis longitudinales sobre el rendimiento en fábrica ya que es 
Ernesto Cavazos Reyes - Violeta Jiménez Zarate - Gil Santana Esparza

uno de los parámetros de la eficiencia del sector azucarero que se monitorea por zafra (CONADESUCA/SAGARPA, 2017), para analizar el comportamiento de diferentes momentos, otra punto que se puede abordar es analizar que innovaciones impactan más en el ISG, tomando como referencia las cuatro temas de investigación e innovación de la agenda nacional de investigación, innovación y transferencia de tecnología de la caña de azúcar que son producción, mejoramiento genético, transformación 0 agroindustria y comercialización (CONADESUCA, 2017a).

\section{REFERENCIAS:}

Adams, R., Jeanrenaud, S., Bessant, J., Denyer, D., \& Overy, P. (2016). Sustainability-oriented Innovation: A Systematic Review. International Journal of Management Reviews, 18(2), 180-205. https://doi.org/10.1111/ijmr.12068

Aguilar-Rivera, N. (2005). Diversificación productiva de la industria azucarera ¿Reto tecnológico, económico, 0 social? Ciecas, 05(18), 54-66. Retrieved from http://www.mundosigloxxi.ciecas.ipn.mx/pdf/v05/18/0 5.pdf

Barbieri, L. A. da rosa, Marques, J. K., Schoproni, R. B., Maffini, C. G., \& Zamberlan, J. F. (2013). The Innovation Process and the Development of a Sustainability Strategy. Latin American Business Review, 14(3-4), 289-308. https://doi.org/10.1080/10978526.2013.837628

Bonsucro. Estándar de Produccion de Bonsucro (2016). Reino unido.

Bossink, B. (2012). Eco-Innovation and Sustainability Management. Routledge. https://doi.org/10.4324/9780203580981

Camisón, C., \& Villar-López, A. (2014). Organizational innovation as an enabler of technological innovation capabilities and firm performance. Journal of Business Research, 67(1), 2891-2902. https://doi.org/10.1016/j.jbusres.2012.06.004

Commission on Environment, W. (1987). Our Common Future: Report of the World Commission on Environment and Development. Retrieved from http://www.un-documents.net/our-common-future.pdf
CONADESUCA/SAGARPA. (2017). Informe estadistico del sector agroindustrial de la caña de azúcar en México, zafras 2006-2007/2015-2016. Retrieved from

https://www.gob.mx/cms/uploads/attachment/file/392 732/Nacional_y_Entidad_Federativa.pdf

CONADESUCA. (2016). Superficie a Industria- lizar Caña a moler Rendimiento de campo Azúcar a producir Rendimiento de fábrica Rendimiento Agroindus- trial. Retrieved

from https://www.gob.mx/cms/uploads/attachment/file/146 567/2doESTIMADODEPRODUCCION_ZAFRA201516.pdf

CONADESUCA. (2017a). Agenda Nacional de Investigación, Innovación y Transferencia de Tecnología de la Caña de Azúcar. Retrieved from https://www.gob.mx/cms/uploads/attachment/file/261 009/Agenda_de_Investigacion_2017.pdf

CONADESUCA. (2017b). Directorio de ingenios azucareros | Comité Nacional para el Desarrollo Sustentable de la Caña de Azúcar | Gobierno | gob.mx. Retrieved November 30, 2017, from https://www.gob.mx/conadesuca/articulos/directoriode-ingenios-azucareros?idiom=es

Cortes, H. G., \& Peña, J. I. (2015). Revista Escuela de Administración de Negocios. Revista Escuela de Administración de Negocios, (78). Retrieved from http://www.redalyc.org/html/206/20640430004/

Dangelico, R. M., Pujari, D., \& Pontrandolfo, P. (2017). Green Product Innovation in Manufacturing Firms: A Sustainability-Oriented Dynamic Capability Perspective. Business Strategy and the Environment, 26(4), 490-506. https://doi.org/10.1002/bse.1932

El Bilali, H. (2018). Relation between innovation and sustainability in the agro-food system. Italian Journal of Food Science, 30, 200-225.

Fajnzylber, F. (1989). Industrialización en América Latina: de la \&quot;caja negra\&quot; al \&quot;casillero vacío\&quot; Retrieved from http://repositorio.cepal.org/bitstream/handle/11362/2 7955/S9000502_es.pdf?sequence=1\&isAllowed=y

FAO. (2017). Trabajo estratégico de la FAO. Trabajo Estratégico de La FAO Para Una Alimentación y Agricultura Sostenibles, 16488, 28. Retrieved from http://www.fao.org/3/a-i6627s.pdf 
ISSN: 1794-9920 - ISSN Electrónico: 2500-9338

Enero - Julio de 2018

Volumen 18 Número 1, Año 2018 Págs. 33 - 43

González-Cortés, M., González-Suárez, E., GonzálezMorales, V., \& Albernas-Carvajal, Y. (2013). Tecnología Química. Tecnología Química (Vol. 33). [publisher not identified]. Retrieved from http://scielo.sld.cu/scielo.php?script=sci_arttext\&pid= S2224-61852013000100003

Instituto Nacional de Estadistica y Geografía. (2014). Conociendo México. Retrieved from http://www.inegi.org.mx/est/contenidos/Proyectos/ce/ ce2014/doc/minimonografias/indazu_ce14.pdf

Jasso-Villazul, J. (2004). Trayectoria tecnológica y ciclo de vida de las empresas: una interpretación metodológica acerca del rumbo de la innovación. Contaduría y Administración UNAM, 49(214), 84-96. Retrieved from http://contaduriayadministracionunam.mx/articulo-3139-17.html

Jasso, J. (1998). De los sistemas nacionales a los supraregionales y subnacionales de innovación. Propuesta analítica y conceptual. Revista de Economia y Empresa, XII(July 1998), 115-131. Retrieved from https://www.researchgate.net/publication/305941594 _De_los_sistemas_nacionales_a_los_supraregional es_y_subnacionales_de_innovacion_Propuesta_ana litica_y_conceptual?_iepl $\% 5$ Bviewld\% $\% 5$ =vrHRINDa U2NFU95nbmkvailK\&_iepl\%5BprofilePublicationltem Variant $\% 5 \mathrm{D}=$ default\&

Jasso, J. (2004). Relevancia de la innovación y las redes institucionales. Aportes, VIII(025), 5-18.

Jasso, J. (2005). La dimensión evolutiva de la innovación: un rumbo necesario de la política científica, tecnológica y de innovación. Economía y Sociedad, 10(15), 99-119.

Jasso, J. (2010). Administracion: innovacion, conceptos, practicas y tendencias. In L. Corona (Ed.), Innovacion ante la sociedad del conocimiento (primera, pp. 215-233). Mexico: Plaza y ValdésUNAM. Retrieved from https://www.researchgate.net/publication/321038375 _Administracion_innovacion_conceptos_practicas_y _tendencias

Jasso, J. (2016). Seguridad productiva en las empresas.pdf. In L. Corona (Ed.), Seguridad productiva en México (Primera, pp. 147-170). México: Miguel Angel Porrúa, Universidad Nacional Autónoma de México. Retrieved from https://www.researchgate.net/publication/305918393
Seguridad_productiva_en_las_empresas

Jasso Villazul, J. (1997). La competitividad internacional empresarial.PDF. ESPACIOS, 18, 21-47. Retrieved from

https://www.researchgate.net/publication/27294321_ La_Competitividad_Internacional_Empresarial_Inten sidad_Patentadora_y_de_Mercado

Lee, H., \& Zhang, H. (2017). Special Issue of Production and Operations Management]: Innovations and Sustainability. Production and Operations Management, 26(4), 757-757. https://doi.org/10.1111/poms.12712

Leff, E. (2000). Tiempo de sustentabilidad. Ambiente \& Sociedade, (6-7), $5-14$. https://doi.org/10.1590/S1414-753X2000000100001

Martins, A. A., Mata, T. M., \& Costa, C. A. V. (2006). Education for sustainability: challenges and trends. Clean Technologies and Environmental Policy, 8(1), 31-37. https://doi.org/10.1007/s10098-005-0026-3

Méndez Chiriboga, M. A. (2012). La sostenibilidad y sustentabilidad en los museos, dos enfoques principales: La museología tradicional y la nueva museología. Estudio de caso en dos museos de la provincia de pichincha, 175. Retrieved from http://repositorio.ute.edu.ec/bitstream/123456789/45 57/1/50939_1.pdf

Mousavi, S., \& Bossink, B. A. G. (2017). Firms' capabilities for sustainable innovation: The case of biofuel for aviation. Journal of Cleaner Production, 167, 1263 1275. https://doi.org/10.1016/j.jclepro.2017.07.146

Nidumolu, R., Prahalad, C. K., \& Rangaswami, M. R. (2009). Why Is Now the Key Driver of Innovation There ' $S$ No Alternative To Sustainable Development . Harvard Business Review, (September), 57-64.

ONU. (2007). Annex 2: Strategy for a climate-neutral UN Objectives. Retrieved from http://www.greeningtheblue.org/sites/default/files/UN ClimateNeutralStrategy.pdf

ONU. (2017). La Agenda de Desarrollo Sostenible Desarrollo Sostenible. Retrieved August 27, 2018, from https://www.un.org/sustainabledevelopment/es/laagenda-de-desarrollo-sostenible/ 
Pérez, C. (1986). LAS NUEVAS TECNOLOGIAS: UNA VISION DE CONJUNTO. Estudios Internacionales. Instituto de Estudios Internacionales Universidad de Chile. https://doi.org/10.2307/41391220

Poorkavoos, M., Duan, Y., Edwards, J. S., \& Ramanathan, R. (2016). Identifying the configurational paths to innovation in SMEs: A fuzzy-set qualitative comparative analysis. Journal of Business Research, 69(12), https://doi.org/10.1016/j.jbusres.2016.04.067

Real Academia de la lengua. (2017). Lista de entradas Diccionario de la lengua española - Edición del Tricentenario. Retrieved October 9, 2018, from http://dle.rae.es/?id=cGmpReY\%7CcGmpuCh\%7Cc GsIrSC

SAGARPA. (2016). REPRESENTA AGROINDUSTRIA CAÑERA NUEVE POR CIENTO DEL PIB PRIMARIO EN ZAFRA 2015-2016. Retrieved November 30, 2017, from http://www.sagarpa.gob.mx/Delegaciones/distritofed eral/boletines/Paginas/JAC0453-10.aspx

Seuring, S., \& Müller, M. (2008). From a literature review to a conceptual framework for sustainable supply chain management. Journal of Cleaner Production, 16(15), 1699-1710.

https://doi.org/10.1016/j.jclepro.2008.04.020

Sun, Z., Hou, J., \& Li, J. (2017). The multifaceted role of information and communication technology in innovation: evidence from Chinese manufacturing firms. Asian Journal of Technology Innovation, 1597(June).

https://doi.org/10.1080/19761597.2017.1302559

Teece, D., \& Pisano, G. (1994). The Dynamic Capabilities of Firms: an Introduction. Retrieved from http://secure.com.sg/courses/lCl/Grab/Reading_Artic les/L03_A02_Teece.pdf

Torres-Vargas, A., \& Jasso-Villazul, J. (2014). Knowledge and quality innovation in the health sector.pdf. In $\mathrm{L}$. Al-Hakim \& C. Jin (Eds.), Quality Innovation. USA: IGI GLOBAL DISSEMINATOR OF KNOWLEDGE. https://doi.org/10.4018/978-1-4666-4769-5.ch008

Torres, A., \& Jasso, J. (2017). Entrepreneurial capabilities and innovation in firms from late industrialising countries: a case study of a Mexican firm. International Journal of Work Innovation, 2(1), 101. https://doi.org/10.1504/IJWI.2017.080724
Torres Vargas, A., \& Jasso Villazul, J. (2016). Handbook of Research on Global Competitive Advantage through Innovation and Entrepreneurship. In L. Al-Hakim, X. Wu, A. Koronios, \& Y. Shou (Eds.), Handbook of Research on Global Competitive Advantage through Innovation and Entrepreneurship (First, Cha, pp. 427-457). Advances in Business Strategy and Competitive Advantage (ABSCA) Book Series, IGI Global. https://doi.org/10.4018/978-1-5225-01350.ch018 Copyright

Tsai, K. H., \& Liao, Y. C. (2017). Sustainability Strategy and Eco-Innovation: A Moderation Model. Business Strategy and the Environment, 26(4), 426-437. https://doi.org/10.1002/bse.1926

ZAFRANET. (2016). Déficit mundial no afecta el abasto azucarero de México: Rello Lara - Zafranet. Retrieved November 30, 2017, from http://www.zafranet.com/2016/06/deficit-mundial-noafecta-el-abasto-azucarero-de-mexico-rello-laral

ZAFRANET. (2018). Caña de azúcar: cultivo agrícola más importante - Zafranet. Retrieved October 8, 2018, from https://www.zafranet.com/2018/04/cana-deazucar-cultivo-agricola-mas-importante/ 